\title{
REGENERACIÓN NATURAL DE Myroxylum balsamun (L) HARMS (ESTORAQUE) EN BOSQUES INTERVENIDOS, SATIPO
}

\section{NATURAL REGENERATION Of Myroxylum balsamun (L) HARMS (ESTORAQUE) IN INTERVENED FORESTS, SATIPO}

\author{
Rubén Gelacio Caballero Salas , Moisés Wilfredo Hurtado León
}

\section{RESUMEN}

El trabajo de investigación se realizó mediante muestreo en dos tipos de bosques de producción forestal, colina alta y baja, ubicadas en las Comunidades Nativas de Gloriabamba y Chamiriari, en la Provincia de Satipo; Región Junín - Perú. Desde hace tres años, en la selva alta, la extracción de la especie, Myroxylum balsamun (L) Harms, conocida como "Estoraque" o "Quina quina", se ha incrementado en forma selectiva y descontrolada. Ante esta problemática fue imprescindible la necesidad de conocer la capacidad de regeneración natural para contribuir con la reposición forestal, evitar la extinción de la especie y efectos lamentables como sucedió con la especie Aniba roseadera, que llegó a desaparecer el material reproductivo y por ende el peligro de extinción. Los objetivos fueron, evaluar la capacidad de regeneración natural luego de la diseminación de semillas hasta la categoría latizal como base de tratamientos silviculturales, comparar los parámetros dasométricos y determinar la cantidad de regeneración por categorías; se llegó a los resultados siguientes: para el tipo de bosque colina alta ubicado sobre los $700 \mathrm{msnm}$, la regeneración natural es nula. Para los bosques de colina baja, ubicado entre 450 a $550 \mathrm{msnm}$, la regeneración natural es de 183 plántulas/ha para el sector de Chamiriari y de 400 plántulas/ha para el sector de Gloriabamba de la categoría brinzal; 100 plantas/ha de la categoría latizal para ambas zonas.

Palabras Clave: Regeneración natural, Myroxylum balsamun, brinzal, latizal .

\begin{abstract}
The investigation work one carries out by means of sampling in two types of forests of forest production, high and low hill, located in the Native Communities of Gloriabamba and Chamiriari, in the province of Satipo; Region Junín - Peru. For three years, in the high forest, the extraction of the species, Myroxylum balsamun (L) Harms, wellknown as "Estoraque" or "Quina quina", it has been increased in selective and uncontrolled form. Before this problem it was indispensable the necessity to know the capacity of natural regeneration to contribute with the forest reinstatement, to avoid the extinction of the species and lamentable effects as Aniba roseadera happened to the species that I arrive it is necessary to disappear the reproductive material and for ende in extinction danger. The objectives were, to evaluate the capacity of natural regeneration after the dissemination of seeds until the category latizal like base of treatments forestry, to compare the parameters dasometric and to determine the quantity of regeneration for categories; you arrives to the following results: for the type of forest high hill located on $700 \mathrm{msnm}$, null of it is of the natural regeneration. For the forests of low hill, located among 400 at $700 \mathrm{msnm}$, the natural regeneration is of 183 plants/ha for the sector of Chamiriari and of 400 plants/ha for the sector of Gloriabamba of the category brinzal; 100 plants/ha of the category latizal for both areas.
\end{abstract}

Key words: Natural regeneration, Myroxylum balsamun, brinzal, latizal.

'Universidad Nacional del Centro del Perú. Facultad de Ciencias Agrarias. 


\section{INTRODUCCIÓN}

Los bosques de la Amazonía, el recurso forestal más importante, ecológica, económica y socialmente, a partir del cual es posible generar miles de empleos forestales, en programas de manejo del recurso existente, reposición forestal, industria maderera, productos forestales no madereros, servicios, bajo una visión expansionista de los mercados (1). Una de las grandes interrogantes que se tienen en la actualidad en relación a los bosques tropicales y sub tropicales del mundo, es la posibilidad de la regeneración natural de especies forestales en ecosistemas primarios como secundarios (2).

El manejo forestal de los bosques intervenidos de Satipo, que suman varios miles de hectáreas, permitirá obtener rendimientos sostenibles asegurando la conservación de los recursos naturales renovables y la biodiversidad en forma permanente, bajo la premisa de que los bosques sigan perdurando como tal, mediante la aplicación de tecnologías forestales adecuadas, como la regeneración natural controlada disminuyendo los impacto que se ejercen sobre el medio ambiente (3). En este sentido, se investigó la regeneración natural de Myroxylum balsamun" (L) Harms, en bosques intervenidos de las Comunidades Nativas de Gloriabamba del distrito del Río Satipo y Chamiriari del distrito de Río Negro; provincia de Satipo, Región Junín.

Los objetivos del estudio fueron: evaluar mediante muestreo diagnostico la capacidad de regeneración natural luego de la diseminación; comparar los parámetros diamétricos entre los tipos de bosque: colina alta y colina baja, y determinar la cantidad de regeneración por categorías.

\section{MATERIAL Y METODOS}

El trabajo se realizó en las comunidades nativas de Gloriabamba y Chamiriari (distrito de Río Tambo, Provincia de Satipo). Con respecto a las zonas de vida, en la primera, predomina el bh-MBT (bosque húmedo montano bajo tropical), de clima "per humedo" con una vegetación propia del bosque de selva alta o bosque de lluvias de montaña, cuyos suelos son acrisoles en una mayor superficie, de color rojizo y parduzco en ciertas partes, productivos cerca de la faja fiscal del río Perené. En la segunda comunidad, predomina el bmh-PT (bosque muy húmedo montano bajo tropical), de clima "húmedo y cálido", con temperatura media anual $23^{\circ} \mathrm{C}$, precipitación anual superior a $2000 \mathrm{~mm}$ por año, con abundancia de especies dominantes, codominantes, intermedios y oprimidos con árboles emergentes de hasta $40 \mathrm{~m}$. de altura. La fauna contiene elementos de selva alta y selva baja, con una población no tan representativa, debido a que los comuneros practicaron la caza de autosostenimiento desde hace 30 años atrás; los suelos del área de manejo son acrisoles, de color rojizo parduzco en ciertas partes.

Se muestreó la regeneración natural (brinzal, latizal y fustal) por tipo de bosque intervenido de colina alta y baja, para evitar la presencia de mayor número de variables; sobre una población de 1000 hectáreas, tomando una muestra de 0,06 ha. en cada comunidad, distribuidas a razón de tres parcelas de 0,01 ha. $(10 \mathrm{~m} \times 10 \mathrm{~m})$ por tipo de bosques. Para el levantamiento de información y evaluaciones se elaboraron formatos por tipo de bosque y categoría de regeneración.

Se evaluó el número de plantas por categorías de regeneración, supervivencia, altura y diámetro, y predominancia de la especie.

\section{RESULTADOS}

Tabla 1. Regeneración natural de Myroxylum halsamun en número de plantas/ha por categoria y por zonas de estudio.

\begin{tabular}{lcccc}
\hline Sector & $\begin{array}{c}\text { Brinzal } \\
\text { plántulas/ha }\end{array}$ & $\begin{array}{c}\text { Latizal } \\
\text { plantas/ha }\end{array}$ & $\begin{array}{c}\text { Fustal () } \\
\text { árboles/ha }\end{array}$ & $\begin{array}{c}\text { Total } \\
\text { plantas/ha }\end{array}$ \\
\hline Gloriabamba & 400 & 100 & 200 & 700 \\
Chamiri & 183 & 100 & 117 & 400 \\
Promedio & 292 & 100 & 159 & 551 \\
\hline
\end{tabular}

(7) Evaluación de árboles fustales como referencia para el estudio.

Tabla 2. Regeneración natural total por categoría en bosques de colina alta en la Comunidad nativa de Gloriabamba. (7).

\begin{tabular}{lrrrrrr} 
& \multicolumn{3}{c}{$N^{0}$ Parcelas } & Total & Ha \\
\cline { 2 - 5 } & $\mathbf{1 0}$ & $\mathbf{4 5}$ & $\mathbf{6 9}$ & Muestra & \\
\hline Brinzal & 15 & 12 & 15 & 42 & 1400 \\
Latizal & 02 & 03 & 02 & 07 & 233 \\
Fustal & 05 & 07 & 06 & 18 & 600 \\
Total & 22 & 22 & 23 & 67 & 2233
\end{tabular}

(\%) Incluye Estoraque. 
La composición de la regeneración natural encontrada en las zonas de estudio, de la especie

Tabla 3. Regeneración natural total por categoria en bosques de colina alta en la Comunidad Nativa de Chamiriari (7).

\begin{tabular}{lccccr}
\hline \multirow{2}{*}{ Categoría } & \multicolumn{3}{c}{ N $^{\circ}$ Parcelas } & \multicolumn{3}{c}{ Total } & Ha \\
\cline { 2 - 5 } & $\mathbf{1 0}$ & $\mathbf{1 4}$ & $\mathbf{2 3}$ & Muestra & \\
\hline Brinzal & 36 & 25 & 19 & 80 & 1333 \\
Latizal & 02 & 00 & 03 & 05 & 83 \\
Fustal & 02 & 08 & 05 & 15 & 250 \\
Total & 40 & 33 & 27 & 100 & 1666
\end{tabular}

(O) Incluye Estoraque.

Tabla 4. Regeneración natural en parcelas de muestreo, por categoría en bosques de colina baja en la Comunidad Nativa de Gloriabamba. (").

\begin{tabular}{lrcccr}
\hline \multirow{2}{*}{ Categoría } & \multicolumn{3}{c}{ N $^{\circ}$ Parcelas } & \multicolumn{3}{c}{ Total } & Ha \\
\cline { 2 - 5 } & $\mathbf{1 0}$ & $\mathbf{4 5}$ & $\mathbf{6 9}$ & Muestra & \\
\hline Brinzal & 15 & 12 & 15 & 42 & 1400 \\
Latizal & 02 & 03 & 02 & 07 & 233 \\
Fustal & 05 & 07 & 06 & 18 & 600 \\
Total & 22 & 22 & 23 & 67 & 2233
\end{tabular}

(7) Incluye Estoraque

Myroxylum balsamun (L) Harms (4), está representada por brinzales procedentes de semillas recientemente germinadas hasta 1,49 m., latizales con alturas que oscilan entre $1,5 \mathrm{~m}$. y 2,99 m. de altura y fustales representados por árboles

de mayor tamaño, de 3,00 m a más, algunos con características de semilleros. Definitivamente no se ha encontrado regeneración natural de Myroxylum balsamun en los tipos de bosque de colina alta (700 a $1200 \mathrm{~m}$.), mientras que para colina baja

existen a razón de 400 y 700 en Chamiriari y Gloriabamba respectivamente (5), distribuidas por

categorías en las tablas 2 y 3 . La diseminación en los dos sitios se presentó entre los meses de agosto y septiembre.

Respecto a la regeneración en general, referida a las especies de valor comercial se tiene los resultados en las tablas 2, 3, 4 y 5 para cada zona en estudio.

Para el caso de Gloriabamba se ha notado mayor presencia de regeneración natural en colina alta de especies como congona Brosimun alicastrum, tulpay Clarisia racemosa, moena amarilla Aniba amazonica., moena blanca Aniba sp., banderilla y roble blanco. Para el caso de colina baja por lo general abundan especies como congona Brosimun alicastrum, estoraque Myroxylum balsamun, Capirona Calycophyllum spruceanum, palo hueso y pochotarque (6)

En la zona de Chamiriari se ha podido observar en colina alta abundancia de moena blanca, amarilla y rosada Aniba sp., tulpay Clarisia racemosa, congona Brosimun alicastrum, catahua Hura crepitans, mientras que en la colina baja se tiene palo hueso, estoraque Myroxylum balsamun, Cedro Cedrela sp., pino chuncho Schizolobium amazonicum y roble colorado como madera corriente (7).

\section{DISCUSION}

Myroxylon es una especie que se encuentra en un rango de altitud de 0 a $1200 \mathrm{msnm}$, prefiere colinas bajas y medias. Es propia de zonas húmedas con una temperatura media anual de $26^{\circ} \mathrm{C}$. Se desarrolla bien cuando la precipitación varía entre 1300 a $4000 \mathrm{~mm} /$ año.

De acuerdo a la clasificación de suelos de la FAO, esta especie prefiere suelos cambisoles, textura franco areno limoso, con un tipo de acidez medianamente ácido ( $\mathrm{pH} 5,7)$ no se desarrolla .

Los bosques productivos de colina alta se ubica en la Comunidad Nativa de Gloriabamba y Chamiriari, a partir de los 700 a 1200 msnm y los de colina baja entre 400 a $700 \mathrm{msnm}$. Se encontraron plantas en germinación de la especie en estudio luego de 25 a

Tabla 5. Regeneración natural en parcelas de muestreo, por categoría en bosques de colina baja en la Comunidad Nativa de Chamiriari. ().

\begin{tabular}{lrcccc}
\hline \multirow{2}{*}{ Categoria } & \multicolumn{3}{c}{ N $^{\circ}$ Parcelas } & \multicolumn{1}{c}{ Total } & Ha \\
\cline { 2 - 5 } & $\mathbf{1}$ & $\mathbf{1 3}$ & $\mathbf{2 2}$ & Muestra & \\
\hline Brinzal & 25 & 14 & 19 & 58 & 967 \\
Latizal & 04 & 02 & 06 & 12 & 200 \\
Fustal & 06 & 06 & 10 & 22 & 367 \\
Total & 35 & 22 & 35 & 92 & 1534
\end{tabular}

*) Incluye Estoraque.

30 días de finalizada la época de diseminación, con un porcentaje de germinación entre 60 a $70 \%$. De acuerdo a la variable analizada de número de plantas de regeneración natural de las categorías brinzal y latizal, los resultados demuestran una mayor cantidad en el orden de 292 plántulas/ha. y 100 plantas/ha. en promedio respectivamente ( 2 y 4). No se encontró regeneración natural de Myroxylum balsamun en el tipo de bosque de colina alta lo cual es corroborado por Angulo (9). En relación a la predominancia de especies diferentes se ha observado mayor cantidad de regeneración natural en la colina baja, 21 y 26 especies comerciales diferentes en Gloriabamba y Chamiriari respectivamente, comparado con 12 y 11 especies en colina alta, debido a las mejores condiciones de calidad de sitio y zonas de vida (8). 


\section{REFERENCIAS BIBLIOGRAFÍCAS}

1. Toledo E. Mercado y desarrollo de la industria forestal con especies de maderas no tradicionales. CIID: Biblioteca: Documentos: Biodiversidad Desarrollo de la Amazonía en una Economía de Mercado. Perú; 2005.

2. Lamprecht H. Silvicutura en los trópicos. Traducción por Dr. Antonio Carrillo. Republica Federal de Alemania. Eschborn; 1990.

3. Gómez A, Vásquez C, Del Amo S, Butanda A. Investigaciones sobre la regeneración de selvas altas en Veracruz". Compañía Editorial Continental S.A. México; 1976.

4. Powell M. Panorama general de los árboles fijadores de nitrógeno - Myroxylon: Bálsamo. Asociación de árboles fijadores de nitrógeno (NFTA). 2004. Hallado en Google:

http//www.winrock.org/forestry/factnet.htm

5. Caballero R. "Plan Operativo Anual Comunidad Nativa de Chamiriari". Satipo Perú; 2002.
6. Bockor I. Análisis de la composición árborea y estructura de un bosque nublado andino. Tesis de Doctorado Universidad de Gottinga Alemania; 1979.

7. Abadie P C. Caracterización del tipo de bosque de terraza en la zona de Genaro Herrera - Iquitos. Tesis. Lima Perú; 1976.

8. FAO. Información y análisis del manejo forestal sostenible, integrando esfuerzos nacionales e internacionales en 13 países tropicales de América Latina". Proyecto GCP/RLA/133/ EC. Chile; 2004; (Fecha de acceso enero 2006) d i s p o n i b l e e $n$ http//www.fao.org/documents/show_cdr.asp

9. Angulo R. W. "Estoraque", Myroxylon balsamun. Estación Experimental Agraria Pucallpa. Consultado 13 de setiembre 2006.

E-mail:satipo10@yahoo.es 